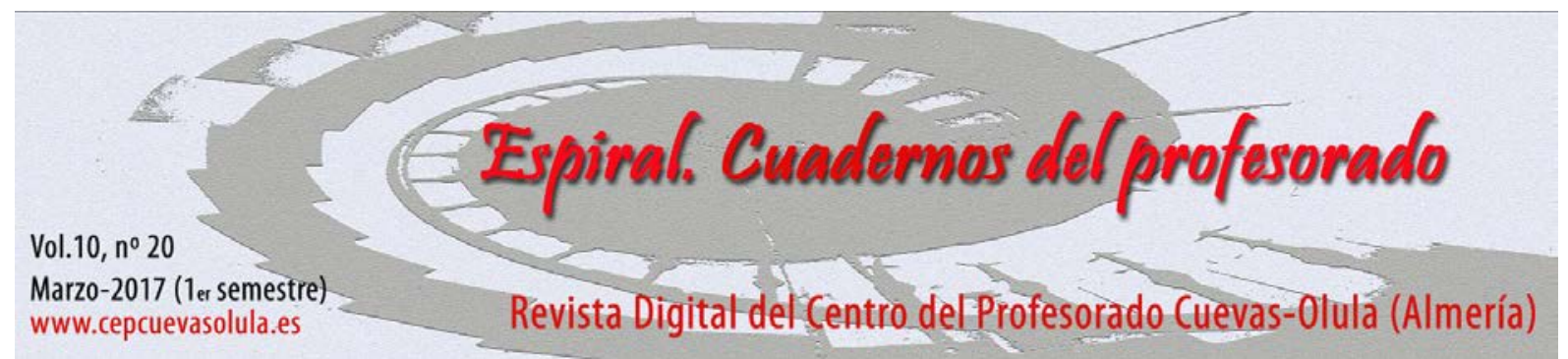

\title{
Satisfacción, motivación y rendimiento académico en estudiantes de Secundaria y Bachillerato: antecedentes, diseño, metodología y propuesta de análisis para un trabajo de investigación
}

\section{Satisfaction, motivation and academic performance in students of secondary and high school: background, design, methodology and proposal of analysis for a research paper}

\author{
Raúl Baños ${ }^{(1)}$, María del Mar Ortiz-Camacho ${ }^{(2)}$, \\ Antonio Baena-Extremera ${ }^{(2)}$, y José Leandro Tristán-Rodríguez ${ }^{(1)}$ \\ ${ }^{(1)}$ Facultad de Organización Deportiva, Universidad Autónoma de Nuevo León, México \\ ${ }^{(2)}$ Facultad Ciencias de la Educación, Universidad de Granada, España
}

RESUMEN: Existen distintas variables contextuales que influyen en la satisfacción, la motivación y el rendimiento académico de los adolescentes en la escuela, dándole importancia a la figura del docente. El objetivo de este trabajo será analizar los perfiles de satisfacción, motivación y el clima de aprendizaje en función del rendimiento académico del discente y de las competencias del profesorado, así como ejemplificar el diseño, metodología y posibles análisis a realizar para responder a los objetivos planteados. La muestra estará compuesta por 890 adolescentes estudiantes de EF (442 hombres y 448 mujeres), con una media de edad de 15.49 años $(D T=1.79)$, pertenecientes a centros públicos y privados de ESO y BACH de la Región de Murcia y la provincia de Alicante (España). El instrumento a utilizar será un cuestionario compuesto por las escalas: Rendimiento académico, Satisfacción con la vida (SATV), Satisfacción con la escuela (ISC), Sport Satisfaction Instrument (SSI), Evaluation of Teaching Competencies Scales (ETCS), Perceptions of Teacher's Emphasison Goals Questionnaire (PTEGQ), Physical Education Classroom Instrument (PECI), Intention to partake in leisure-time physical activity, Importancia y utilidad de la Educación Física (IEF). En este artículo presentamos el diseño de la investigación. Pensamos que los resultados obtenidos tendrán una gran repercusión en el sistema educativo español e incitarán a reflexionar sobre diferentes aspectos del currículo en la ESO y BACH.

Palabras clave: satisfacción, rendimiento académico, competencias docentes, secundaria, Educación Física.

ABSTRACT: There are several contextual variables that influence the satisfaction, motivation and academic performance of adolescents in school, giving importance to the teacher figure. The aim of this work will be to analyze the satisfaction profiles, motivation and the learning climate in function of the academic performance of the student and the competition of the teachers as well as to exemplify the design, methodology and possible analysis to be carried out in order to respond to the stated objectives. The sample will be composed of $890 \mathrm{PE}$ students (442 men and 448 women), with a mean age of 15.49 years $(S D=1.79)$ belonging to public and private ESO and BACH centers in the Region of Murcia and Alicante. The instrument to be used will be a questionnaire composed by the scales: Academic Performance, Satisfaction with Life (SATV), Satisfaction with School (ISC), Evaluation of Teaching Competencies Scales (ETCS), Perceptions of Teachers Emphasis on Goals Questionnaire (PTEGQ), Physical Education Classroom Instrument (PECI), Intention to Partake in Leisure-Time Physical Activity, Importance and Utility of Physical Education (IEF). In this article we present the design of the research. We believe that the results obtained will have a great impact on the Spanish educational system and will incite to reflect on different aspects of the curriculum in secondary and high school.

Key words: satisfaction, academic performance, teaching competences, secondary, physical education. 
Baños, R., Ortiz-Camacho, M. M., Baena-Extremera, A., y Tristán-Rodríguez, J. L. (2017). Satisfacción, motivación y rendimiento académico en estudiantes de Secundaria y Bachillerato: antecedentes, diseño, metodología y propuesta de análisis para un trabajo de investigación. Espiral. Cuadernos del Profesorado, 10(20), 40-50. Disponible en: http://espiral.cepcuevasolula.es/

Fecha de recepción: 02/07/2016

Fecha de aceptación: 11/01/2017
Enviar correspondencia a: raulfb89@gmail.com

\section{Introducción}

La investigación sobre educación genera un importante interés donde examinar los posibles factores tanto del proceso de enseñanza como del proceso de aprendizaje. Entre ellos, el rendimiento académico y el crecimiento personal del estudiante se transforman en aspectos fundamentales del proceso instruccional a tener en cuenta por el docente. Variables como la satisfacción, el comportamiento, la motivación de los adolescentes e incluso las competencias docentes, son grandes influyentes en un efectivo proceso de enseñanza-aprendizaje en la Educación Primaria, Secundaria Obligatoria (ESO) y en el Bachillerato (BACH).

El rendimiento académico es entendido como "la productividad del sujeto, el producto final de la aplicación de su esfuerzo, matizado por sus actividades, rasgos y la percepción más o menos correcta de los cometidos” (Muela, García-León, Augusto, \& López-Zafra, 2010). Es una variable que preocupa en el sistema educativo español, sobre todo después de los últimos resultados obtenidos en el Programa Internacional para la Evaluación de Estudiante (Informe PISA) (Informe PISA, 2016). Esta preocupación se traduce en los últimos años en multitud de estudios que versan sobre cómo mejorar el rendimiento de los discentes (Fajardo, Maestre, Felipe, León, \& Polo, 2017).

Por ejemplo, en los últimos años los investigadores han analizado las influencias en el rendimiento y en el aprendizaje escolar según el contexto que engloba al centro, estudiando diferentes variables como las competencias docentes, los comportamientos disruptivos de los estudiantes, la motivación, la práctica deportiva en su tiempo libre e incluso el bienestar del discente con la vida, con la escuela y con las diferentes asignaturas (Danielsen, Samdal, Hetland, \& Wold, 2009; EstebanCornejo et al., 2014; Lewis, Huebner, Malone, \& Valois, 2011; Moloi, 2010; Moreno, 2009; Robles, Solbes, Cantó, \& Lozano, 2015; Suldo \& Huebner, 2006).

En el estudio de estas variables, la teoría del bienestar subjetivo podría contribuir de forma positiva a esclarecer diversos comportamientos del discente. Sobre esta teoría, Diener (2009) destaca dos componentes importantes de este constructo; uno cognitivo (satisfacción con la vida), entendiéndose como el conjunto de toda la vida propia del discente, y otro afectivo (balance afectivo), siendo el resultado de las reacciones inmediatas y continuas a los sucesos que le conciernen al estudiante. Éste último a su vez, se divide en un balance afectivo positivo y negativo (Diener, Lucas, \& Oishi, 2002). La satisfacción con la escuela es una variable determinante en el progreso del estudiante que influye en las relaciones sociales y rendimiento escolar (Danielsen et al., 2009), en los niveles de estrés (Hui \& Sun, 2010), en el compromiso del discente con su trabajo escolar (Lyons \& Huebner, 2016) y en la práctica deportiva extraescolar (Takakura, Wake, \& Kobayashi, 2010). El hecho de que el estudiante se sienta satisfecho, divertido y a gusto (Baena-Extremera \& Granero Gallegos, 2015), contribuirá a reducir el abandono escolar y las conductas disruptivas (Elmore \& Huebner, 2010), contribuyendo además de manera positiva en la satisfacción con la vida del estudiante (Lewis et al., 2011).

En esta línea, se ha demostrado cómo la satisfacción/diversión en la asignatura de Educación Física (EF) predice positivamente la satisfacción/diversión con la escuela (Baena-Extremera \& Granero-Gallegos, 2015). Se conoce también como repercuten las materias de Inglés y Lengua en el rendimiento académico de los estudiantes (Kofou, 2015; Yeung, Lau, \& Nie, 2011), demostrándose que aquellos alumnos que encuentran interesantes dichas materias y se sienten satisfechos en sus 
clases, se comprometen con la realización de las tareas, mostrando menos conductas de desapego y aumentando su rendimiento académico (Rotgans \& Schmidt, 2011; Yeung, Lau, \& Nie, 2011). Por otro lado, los altos niveles de aburrimiento en la materia de Matemáticas aumentan la probabilidad de abandono escolar aunque no se relaciona con el rendimiento académico (Mora, 2012). Este dato, contrapone otros trabajos, donde sí se ha encontrado relación entre el rendimiento académico y la insatisfacción con las materias de Historia y Matemáticas (Polikhun, 2010). Teniendo en cuenta lo anterior, sería muy interesante para el mundo académico profundizar en cómo afecta cada una de las asignaturas del currículo de Secundaria en la satisfacción con la escuela y, a su vez, con las calificaciones. Quizás, con estos resultados, podríamos resolver preguntas tan interesante como si, ¿todas las materias afectan por igual a la satisfacción escolar?, ¿algunas más que otras?, ¿dependerá de las horas de clase, del profesor, de la metodología, o de otros factores?

Por otro lado, la figura del docente adquiere un papel importante tanto en la satisfacción del estudiante como en el aprendizaje de éste, resultando interesante investigar sobre qué competencias son las que debe reunir un buen profesor, mejorando así la intervención docente. El profesorado debe desarrollar una serie de habilidades entre las que destaca ser perfeccionista, atento, alentador y cariñoso, pero sobre todo que tenga la capacidad de divertir, entusiasmar, ser afectivo, abierto y comprensivo (Moreno, 2009). Autores como Smith y Suzuki (2015), además afirman que el docente debe ser capaz de crear distintos ambientes de aprendizaje en función de las características del aula de secundaria, con novedosos recursos didácticos. Sin embargo algunos de éstos afirman sentirse satisfechos con las metodologías tradicionales, desinteresándose por los nuevos paradigmas educativos (Glasnović \& Domović, 2009) y las nuevas metodologías. Este desinterés de los docentes con la enseñanza produce un impacto negativo en los estudiantes (Cameron \& Lovett, 2015), que se traduce en bajo compromiso escolar y rendimiento académico. Desde el área de la EF, esto repercutirá en un mayor sedentarismo de los adolescentes acompañado de los efectos perjudiciales que conllevan para la salud (Rué \& Serrano, 2014).

En relación al discente, dos teorías que nos pueden aportar un gran avance para esta futura investigación, son la Teoría de la Acción Razonada (Ajzen \& Fishbein, 1980) y la Teoría del Comportamiento Planeado (Ajzen, 1991). Según estos autores, la primera teoría predice el comportamiento debido a que la conducta se encuentra bajo un control voluntario, la intención no cambia antes de que el comportamiento sea observado, porque intenciones y comportamiento están vinculados a los objetivos y contexto en el que se encuentre el sujeto. Se considera la "intención" como la mayor predicción en las acciones futuras de ejercicio físico (Ajzen \& Fishbein, 1980). En relación a la intención de práctica física y su realización, Oviedo et al. (2013) hallaron que la mayoría de los adolescentes españoles dedican poco tiempo a la realización de la actividad física, obteniendo las mujeres mayores niveles de sedentarismo. Una forma de aumentar la intención de práctica de actividad física en el tiempo libre de los adolescentes es que el docente adopte en las clases de EF estrategias de motivación que ayuden a modificar su conducta, tal y como nos aporta Taylor, Ntoumanis, Standage, y Spray (2010). Esta falta de práctica deportiva está relacionada con la baja importancia que el estudiante dé a la asignatura de EF (Baena-Extremera, Granero-Gallegos, SánchezFuentes, \& Martínez-Molina, 2014), relacionándose con bajos niveles de actividad físico-deportiva durante el tiempo libre (Kilpatrick, Hebert, \& Jacobsen, 2002). Por este motivo, es interesante indagar sobre cómo afecta el papel del profesorado de EF en la intención de práctica deportiva futura, así como en la importancia que el estudiante otorga a la asignatura. Teniendo en cuenta esto, hay que destacar que la Organización Mundial de la Salud (OMS) (2014) ya alertó años atrás que la inactividad física ocupaba el cuarto lugar entre los principales factores de riesgo de mortalidad a nivel mundial, con una cifra aproximada de 3,2 millones de muertes por año. Los niños y adolescentes obesos tienen más probabilidades de desarrollar una serie de problemas de salud en la edad adulta como cáncer (endometrio, mama y colon) resistencia a la insulina, cardiopatía o artrosis a causa del sedentarismo (OMS, 2016). De hecho, son alarmantes las cifras de 42 millones de niños que sufren sobrepeso u obesidad, pudiendo aumentar a 70 millones para 2025 (OMS, 2016) y, la EF en general y los docentes en particular, podrían tener un gran papel en este remedio. 
Acorde con lo mencionado en el anterior párrafo, la teoría de metas de logro de Nicholls (1989) aporta información relevante sobre qué ambientes de aprendizaje son los más idóneos para que los estudiantes se motiven, practiquen actividad físico-deportiva en su tiempo libre, se sientan satisfechos e incluso tengan un comportamiento ejemplar en EF (Granero-Gallegos, Baena-Extremera, Pérez-Quero, Ortiz-Camacho, \& Bracho-Amador, 2012; Krech, Kulinna, \& Cothran, 2010; Moreno, González-Cutre, \& Ruiz, 2009). El anterior constructo teórico, analiza cómo los factores ambientales y disposicionales influyen en los entornos de logro en los cuales actúan las personas en diferentes contextos de su vida motivados por la obtención del éxito. De este modo, en la asignatura de EF se pueden producir diversos conflictos en la obtención del éxito en las tareas, ocasionados por ejemplo por el poco dominio de las habilidades motrices del alumnado o incluso por la búsqueda obsesiva de la victoria de muchos estudiantes (Klomsten, Marsh, \& Skallvick, 2005). Igualmente, la asignatura de EF es propicia para crear un clima de diversión y evasión del estrés, dentro de clase. Pero, estos factores, ¿podrían estar condicionados por el género del docente?, ¿los profesores y profesoras tienden a crear el mismo clima de clase y por consiguiente, las mismas conductas disruptivas en los alumnos?, ¿tanto ellos como ellas, afectan por igual a la intención de práctica física y la importancia concedida a esta materia?, ó ambos géneros, ¿afectan por igual al rendimiento académico? Teniendo en cuenta estas cuestiones, sería interesante conocer si el género del profesor de EF ejerce alguna influencia sobre la importancia concedida a esta asignatura, el clima motivacional percibido por los alumnos, sus conductas disruptivas y la intención de práctica física futura.

A tenor de lo anteriormente expuesto, creemos que sería de gran interés para la comunidad educativa y científica responder a las preguntas anteriormente señaladas. Para ello, describimos a continuación el diseño del futuro trabajo de investigación.

\section{Método}

\section{Objetivos del Estudio}

A continuación se describen los objetivos fundamentales del trabajo que se pretende llevar a cabo:

1. Analizar la predicción de diferentes asignaturas del currículum, en relación a la satisfacción intrínseca en la escuela y a la mejora del rendimiento académico.

2. Analizar como las competencias del docente de EF pueden predecir la satisfacción con la escuela, y éstas a su vez, la satisfacción con la vida en el alumnado de educación secundaria.

3. Conocer si existe efecto del género del profesor de Educación Física sobre el rendimiento académico, la importancia concedida a esta asignatura, el clima motivacional percibido por los alumnos, sus conductas disruptivas y la intención de práctica física futura.

\section{Diseño y Muestra}

El diseño que planteamos para la consecución de los objetivos es de carácter no experimental, seccional y descriptivo (Sierra, 2011). El diseño muestral será no probabilístico y por conveniencia según la posibilidad de acceso a él, seleccionando un total de 890 adolescentes estudiantes de EF (442 hombres y 448 mujeres). La edad media es de 15.49 años $(\mathrm{DT}=1.79$ ), perteneciendo a cinco centros públicos y privados de ESO y BACH de la Región de Murcia y de la provincia de Alicante (España). El rango en función del curso académico de la muestra está comprendido entre estudiantes de $1^{\circ}$ de ESO y $2^{\circ}$ de BACH. El $17.1 \%$ de la muestra pertenece a $1^{\circ}$ de ESO, un $18.0 \%$ a $2^{\circ}$ de ESO, el $20.4 \%$ a $3^{\circ}$ de ESO, un $20.9 \%$ a $4^{\circ}$ de ESO, un $11.3 \%$ a $1^{\circ}$ de BACH y por último, un $12.2 \%$ a $2^{\circ}$ de BACH.

\section{Instrumentos}

Para llevar a cabo esta investigación, se elaborará un cuestionario compuesto de las siguientes escalas o instrumentos: 
1. Rendimiento académico. Para evaluar el rendimiento académico de los estudiantes se confeccionará un cuestionario con cada una de las materias encuestadas. Las respuestas se recogerán con una escala tipo Likert desde el 1 (la calificación más baja), hasta 10 (la calificación más alta), tal como se evalúa a los estudiantes.

2. Satisfacción con la vida. Para evaluar la satisfacción con la vida se utilizará un instrumento traducido al castellano por Atienza, Pons, Balaguer y García-Merita (2000) de la versión inglesa (SWLS) de Diener, Emmons, Larsen y Griffin (1985). Este instrumento presenta 5 ítems, recogiéndose las respuestas con una escala tipo Likert desde 1 (muy en desacuerdo) hasta 5 (muy de acuerdo). Los ítems son: 1) En la mayoría de los aspectos mi vida es como quiero que sea, 2) Hasta ahora he conseguido de la vida las cosas que considero importantes, 3) Estoy satisfecho con mi vida, 4) Si pudiera vivir mi vida otra vez, la repetiría tal y como ha sido, y 5) Las circunstancias de mi vida son buenas.

3. Satisfacción con la escuela. Se empleará el Cuestionario de Satisfacción con la Escuela validado por Castillo, Balaguer y Duda (2001) del Intrinsic Satisfaction Classroom Scale (ISC) (Duda \& Nicholls, 1992; Nicholls, 1989). Este instrumento presenta ocho ítems que miden el grado de satisfacción con la escuela con dos subescalas, cinco ítems que miden la satisfacción/diversión y tres ítems que miden el aburrimiento. La escala está precedida por la frase "Dinos tu grado de desacuerdo o acuerdo en relación a las siguientes afirmaciones, referidas a todas tus clases en el instituto", continuada por los ochos ítems. Un ejemplo de ítem de la subescala de satisfacción/diversión es "Normalmente disfruto aprendiendo en el colegio". Un ejemplo de ítem de aburrimiento es "Normalmente deseo que se acabe rápidamente el día de colegio". Las respuestas se recogerán mediante una escala tipo Likert que oscila entre 1 (muy en desacuerdo) y 5 (muy de acuerdo).

4. Sport Satisfaction Instrument (SSI) adaptada a Educación Física. Se usará la versión española (Balaguer, Atienza, Castillo, Moreno, \& Duda, 1997) del Sport Satisfaction Instrument (SSI) de Duda y Nicholls (1992), adaptada a EF (SSI-EF) (Baena-Extremera, Granero-Gallegos, Bracho-Amador, \& Pérez-Quero, 2012). El SSI-EF consta de 8 ítems para medir la satisfacción intrínseca en una actividad deportiva mediante dos subescalas que miden satisfacción/diversión (5 ítems) y aburrimiento (3 ítems) en la práctica deportiva. La escala está precedida por la frase "Según la asignatura que doy en clase...", haciendo referencia a las distintas materias recibidas por los estudiantes (Lengua, Matemáticas, Educación Física, Inglés y Geografía e Historia). Un ejemplo de ítem de satisfacción/diversión es "Normalmente encuentro la asignatura de... interesante» y, de aburrimiento es "En las clases de... a menudo sueño despierto en vez de pensar en lo que hago realmente". Las respuestas se recogerán en una escala de tipo Likert de 5 puntos que oscila desde 1 (muy en desacuerdo) a 5 (muy de acuerdo).

5. Evaluation of Teaching Competencies Scales (ETCS). Este instrumento, adaptado al castellano de la versión inglesa de Catano y Harvey (2011), presenta nueve ítems que miden las percepciones de los estudiantes sobre la eficacia de la docencia. La versión castellana fue adaptada para medir las competencias del profesorado de Educación Física (BaenaExtremera, Granero-Gallegos, \& Martínez-Molina, 2015). Las respuestas se recogerán en una escala de ítems politómicos de siete puntos que oscila entre los valores Bajo $(1,2)$, Medio $(3,4,5)$ y Alto $(6,7)$. Los ocho constructos a medir son la comunicación, conciencia de trabajo, creatividad, feed-back, consideración individual del alumno/a, profesionalidad, resolución de problemas, y conciencia social.

6. Perceptions of Teacher's Emphasis on Goals Questionnaire (PTEGQ). Se utilizará la versión española (Ruiz-Juan, 2014) de la versión inglesa PTEGQ de Papaioannou,Tsigilis, Kosmidou y Milosis (2007). Este instrumento presenta 24 ítems que miden las percepciones que tienen los alumnos de sus profesores de EF. Está compuesto por cuatro dimensiones: Maestría (6 ítems), Rendimiento-aproximación (6 ítems), Rendimiento- 
evitación (6 ítems) y Aprobación social (6 ítems). La escala está precedida por la frase “Mi profesor de Educación Física...”. Las respuestas se recogerán en escala tipo Likert desde 1 (totalmente en desacuerdo) hasta 5 (totalmente de acuerdo). Un ejemplo de ítem de maestría es "Está muy feliz cuando aprendo nuevas habilidades o juegos", de rendimiento-aproximación "Anima a los alumnos a jugar mejor que los demás", de rendimiento-evitación "A menudo hace que me preocupe por si me dice que no soy competente o capaz en la clase de Educación Física", y de aprobación social "Pretende que yo aprenda habilidades o juegos para que mis compañeros me valoren"

7. Physical Education Classroom Instrument (PECI). Se utilizará la versión de GraneroGallegos y Baena-Extremera (2016) adaptado a Educación Física. Este instrumento está compuesto por 20 ítems que miden las percepciones que tienen los estudiantes sobre su comportamiento en las clases de Educación Física. Está compuesto por cinco dimensiones: agresividad (4 ítems), irresponsabilidad y bajo compromiso (4 ítems), desobediencia de las normas (4 ítems), perturbador del ambiente de clase (4 ítems) y bajo autocontrol personal (4 ítems). La escala está precedida por la frase "Piensa en tu propio comportamiento en clase de Educación Física y dinos tu grado de desacuerdo o acuerdo...”. Las respuestas se recogerán en escala tipo Likert desde 1 (muy en desacuerdo) hasta 5 (muy de acuerdo). Un ejemplo de ítem de agresividad es "Amenazo a los demás compañeros/as de clase", de irresponsabilidad y bajo compromiso "Soy perezoso en clase", de desobediencia de las normas "Interrumpo las clases", de perturbador del ambiente de clase "No presto atención en clase de Educación Física”, y de bajo autocontrol personal "Soy peleón".

8. Intention to partake in leisure-time physical activity. Se utilizará la versión castellana (Granero-Gallegos et al., 2014), traducida de la versión inglesa (Chatzisarantis, Biddle, \& Meek, 1997) que está basado en la obra de Ajzen y Madden (1986). El instrumento trata de evaluar la intención de los estudiantes de ser físicamente activos en su tiempo libre durante el período de un mes. La escala está precedida por la frase "En mi tiempo libre, fuera del instituto...”. Las respuestas se recogerán en escala tipo Likert desde 1 (totalmente en desacuerdo) hasta 7 (totalmente de acuerdo). Un ejemplo de ítem es «Estoy decidido a hacer ejercicio físico».

9. Importancia y utilidad de la EF (IEF). Dicho instrumento mide la importancia y utilidad concedida por el alumnado a la Educación Física (Moreno et al., 2009) a través de 3 ítems. Los alumnos responderán en una escala tipo Likert de 4 puntos que va desde 1 (totalmente en desacuerdo) y 4 (totalmente de acuerdo). La escala está precedida por la frase "En mis clases de Educación Física...”. Las respuestas se recogen en escala tipo Likert desde 1 (totalmente en desacuerdo) hasta 4 (totalmente de acuerdo).Un ejemplo de ítem es "Considero importante recibir clase de Educación Física".

\section{Procedimiento}

Se pedirá permiso previo a los órganos competentes para poder llevar a cabo esta investigación. Se informará con detalle a padres/tutores y adolescentes acerca del protocolo y objetivos del estudio, de la voluntariedad para participar y de la confidencialidad de las respuestas y datos, siendo un registro indispensable la firma del consentimiento informado por parte de ambos. Los instrumentos para medir las diferentes variables se administrarán en el aula por los propios investigadores y sin la presencia del docente, dejando un tiempo de entre 30 y 45 minutos. Se les informará de que no habrá respuestas correctas o incorrectas, y se les pedirá al comienzo que contesten con la máxima sinceridad y honestidad. Si surgen dudas por parte de los adolescentes durante el trabajo de campo, se resolverán en ese momento por el equipo de investigadores.

\section{Análisis de los Datos}



siguiente.

Para llevar a cabo este trabajo, el planteamiento de análisis estadístico debe pasar por lo

En relación al primer objetivo se llevará a cabo un análisis factorial confirmatorio (AFC) de la escala de Satisfacción con cada una de las asignaturas, así como con la escala de satisfacción intrínseca con la escuela. Se calcularán varios índices de fiabilidad y validez, como el alfa de Cronbach, la fiabilidad compuesta y la varianza media extraída (AVE) para cada instrumento. A continuación, se llevará a cabo análisis de correlaciones de cada escala de satisfacción adaptada a cada asignatura, con la satisfacción escolar y la nota académica. Posteriormente, se llevará a cabo diversos modelos de ecuaciones estructurales diferenciando por asignatura, para responder al objetivo de este trabajo. Los cálculos se llevaron a cabo con el paquete estadístico SPSS v.22 y LISREL 8.80.

En el segundo objetivo se llevará al igual que el primero, un AFC para estudiar la adecuación de dichos instrumentos a las muestras utilizadas en esta investigación, seguidos de los cálculos de índices de fiabilidad y validez (alfa de Cronbach, fiabilidad compuesta y AVE) para cada instrumento. A continuación, se realizará un análisis de correlaciones entre los instrumentos utilizados. Posteriormente, se llevarán a cabo diversos modelos de ecuaciones estructurales, para responder al objetivo de este trabajo. Los cálculos deberán procesarse con el paquete estadístico SPSS v.22 y LISREL 8.80.

Para finalizar con el tercer objetivo, se llevarán a cabo AFC para estudiar la adecuación de dichos instrumentos a la muestras a utilizar. Se calcularán como en casos anteriores la fiabilidad y validez de cada instrumento. Seguidamente, se procederá a realizar un Modelo Lineal de Regresión Multinivel, estudiando la asociación del género del docente con las dimensiones de la importancia de la EF, el clima motivacional percibido por el alumno, las conductas disruptivas, la intención de práctica física y rendimiento académico, todo ello ajustado por al tipo de centro, a la edad, al género del alumnado y al grupo clase al que pertenecían. Todos los cálculos de este objetivo se llevaron a cabo con el paquete estadístico SPSS v.22 y LISREL 8.80 .

\section{Resultados y Discusión}

El realizar un proyecto de investigación de esta relevancia supondrá obtener unos resultados que puedan generar una gran repercusión en el sistema educativo español, con la consiguiente reflexión sobre determinados aspectos del currículo de ESO y BACH. Con la obtención de estos resultados se espera concienciar al profesorado sobre nuevas formas de planificar y organizar el aula, tanto de los niveles educativos mencionados como el de las propias asignaturas. De este modo, los docentes conocerán como deben estructurar sus tareas en el aula y cómo repercutir en los adolescentes con estilos de vida más activos.

El validar la escala de satisfacción con la EF (Baena-Extremera et al., 2012) en otras materias de ESO y BACH, servirá para que los docentes obtengan información sobre si los estudiantes se están aburriendo o divirtiendo en sus clases. Esto es importante para prevenir el absentismo en las aulas y el abandono escolar ya que, si un alumno encuentra la enseñanza monótona y aburrida, la posibilidad de que deserte de la escuela se incrementaría (Elmore \& Huebner, 2010), y estas cifras, desde hace años, preocupan en el sistema educativo español (Ministerio de Educación, Cultura y Deporte, 2013). Sobre esto, se ha demostrado que un estudiante que se divierte y se siente satisfecho predice la satisfacción con la escuela (Baena-Extremera \& Granero-Gallegos, 2015), la satisfacción con la vida (Lyons \& Huebner, 2016), y el rendimiento académico (Paulsen \& Feldman, 2005). Conocer como predice cada una de las asignaturas la satisfacción y el rendimiento académico puede ayudar a nuestros órganos gubernamentales a crear leyes educativas que se asemejen más a la realidad, mejorando así el rendimiento académico y haciendo más eficiente las grandes inversiones económicas que se realizan en educación (OCDE, 2013).

En relación a las competencias docentes y cómo influyen éstas en el estudiante, los resultados del presente trabajo podrían revelar resultados interesantes sobre cómo el profesorado se comunica, retroalimenta, resuelve los problemas y conciencia del trabajo a los estudiantes (Catano \& Harvey, 
2011), en definitiva, conocer si las competencias de éste, consiguen altos niveles de satisfacción en la escuela y en la vida del estudiante. También estudiar cómo influye el género del docente sobre los comportamientos negativos y la importancia que el estudiante concede a la materia de EF pueden aportarnos resultados relevantes, ya que existen indicios que las profesoras suelen ser más víctimas de las conductas negativas de los discentes (Cothran \& Kulinna, 2007). En esta línea, despierta gran interés averiguar que climas de aprendizaje creados por el docente en sus aulas son más favorables para conseguir un mayor compromiso, una mayor adherencia a la práctica deportiva, y un mayor esfuerzo y satisfacción con la vida en el alumnado (Moreno, Cervelló, \& González-Cutre, 2007; Conley, Pintrich, Vekiri, \& Harrison, 2004; Sproule, Wang, Morgan, McNeill, \& McMorris, 2007; Standage, Duda, \& Ntoumanis, 2003).

Para finalizar, con este estudio esperamos revelar resultados que ayuden a los docentes de EF a conocer qué ambientes de aprendizaje promueven estilos de vida más activos, teniendo en cuenta el problema del sedentarismo en España (Román et al., 2006). De este modo, en función de cómo el profesorado motive en las clases de EF aumentará la intención de práctica de ejercicio físico en el tiempo libre (Hagger, Chatzisarantis, Culverhouse, \& Biddle, 2003), así como la importancia y utilidad que el estudiante dé a la EF (Baena-Extremera et al., 2014). Los resultados del presente proyecto de investigación sin duda, pueden llegar a ser de gran relevancia en la organización de la docencia y en la preparación del profesorado actual y futuro.

\section{Referencias}

Ajzen, I. (1991). The theory of planned behaviour. Organizational Behaviour and Human Decision Processes, 50, 179-211.

Ajzen, I. y Fishbein, M. (1980). Understanding Attitudes and Predicting Social Behaviour. Englewood-Cliffs, NJ: Prentice-Hall.

Ajzen, I. y Madden, T. (1986). Prediction of goal-directed behaviour: Attitudes, intentions and perceived behavioural control. Journal of Experimental Social Psychology, 22, 453-474.

Atienza, F. L., Pons, D., Balaguer, I., y García-Merita, M. (2000). Propiedades psicométricas de la Escala de Satisfacción con la Vida en adolescentes. Psicothema, 12(2), 314-319.

Baena-Extremera, A. y Granero-Gallegos, A. (2015). Modelo de predicción de la satisfacción con la educación física y la escuela. Revista de Psicodidáctica, 20(1), 177-192.

Baena-Extremera, A., Granero-Gallegos, A., Bracho-Amador, C., y Pérez-Quero, F. J. (2012). Versión española del Sport Satisfaction Instrument (SSI) adaptado a la educación física. Revista de Psicodidáctica, 17(2), 377-396.

Baena-Extremera, A., Granero-Gallegos, A., Sánchez-Fuentes, J. A., y Martínez-Molina, M. (2014). Modelo predictivo de la importancia y utilidad de la Educación Física. Cuadernos de Psicología del Deporte, 14(2), 121-130.

Baena-Extremera, A., Granero-Gallegos, A., y Martínez-Molina, M. (2015). Validación española de la Escala de Evaluación de la Competencia Docente en Educación Física de secundaria. Cuadernos de Psicología del Deporte, 15(3), 113-122.

Balaguer, I., Atienza, F. L., Castillo, I., Moreno, Y., y Duda, J. L. (1997). Factorial structure of measures of satisfaction/interest in sport and classroom in the case of Spanish adolescents. En 4th European Conference of Psychological Assessment (Vol. 76).

Cameron, M. y Lovett, S. (2015). Sustaining the commitment and realizing the potential of highly promising teachers. Teachers and Teaching, 21(2), 150-163.

Castillo, I., Balaguer, I., y Duda, J. L. (2001). Perspectivas de meta de los adolescentes en el contexto académico. Psicothema, 13(1), 79-86.

Catano, V. M. y Harvey, S. (2011). Student perception of teaching effectiveness: development and validation of the Evaluation of Teaching Competencies Scale (ETCS). Assessment y Evaluation in Higher Education, 36(6), 701-717.

Chatzisarantis, N. L., Biddle, S. J., y Meek, G. A. (1997). A self-determination theory approach to the study of intentions and the intention-behaviour relationship in children's physical activity. British Journal of Health Psychology, 2(4), 343-360. 
Conley, A. M., Pintrich, P. R, Vekiri, I., y Harrison, D. (2004). Changes in epistemological beliefs in elementary science students. Contemporary Educational Psychology, 29, 186-204.

Cothran, D. J. y Kulinna, P. H. (2007). Students' reports of misbehavior in physical education. Research Quarterly for Exercise and Sport, 78, 216-224.

Danielsen, A. G., Samdal, O., Hetland, J., y Wold, B. (2009). School-related social support and students' perceived life satisfaction. Journal of Education Research, 102(4), 303-318.

Diener, E. (2009). Assessing well-being: Progress and opportunities. En E. Diener (Ed.), Assessing well-being. The collected works of Ed Diener (pp. 25- 65). New York: Springer (Social Indicators Research Series, 39).

Diener, E., Emmons, R., Larsen, R. J., y Griffin, S. (1985). The Satisfaction With Life Scale. Journal of Personality Assessment, 49, 71-75.

Diener, E., Lucas, R. E., y Oishi, S. (2002). Subjective well-being: The science of happiness and life satisfaction. En C. R. Snyder y S. Lopez (Eds.), Handbook of positive psychology (pp. 463-473). London: Oxford University Press.

Duda, J. L. y Nicholls, J. G. (1992). Dimensions of achievement motivation in schoolwork and sport. Journal of Educational Psychology, 84(3), 290-299.

Elmore, G. M. y Huebner, E. S. (2010). Adolescents satisfaction with school experiences: Relationships with demographics, attachment relationships, and school engagement behavior. Psychology in the Schools, 47(6), 525-537.

Esteban-Cornejo, I., Tejero-González, C. M., Martínez-Gómez, D., del-Campo, J., González-Galo, A., PadillaMoledo, C., ... y UP y DOWN study group. (2014). Independent and combined influence of the components of physical fitness on academic performance in youth. The journal of pediatrics, 165(2), 306-312.

Fajardo, F., Maestre, M., Felipe, M. E., León, B., y Polo, M. I. (2017). Análisis del rendimiento academic de los alumnus de educación secundaria obligatoria según las variables familiars. Educación XX1: Revista de la Facultad de Educación, 20(1), 209-232.

Glasnović, D. y Domović, V. (2009). Using maths textbooks in lower secondary education. Odgojneznanosti, 11(2 (18), 45-65.

Granero-Gallegos, A. y Baena-Extremera, A. (2016). Validación española de la versión corta del Physical Education Classroom Instrument para la medición de conductas disruptivas en alumnado de secundaria. Cuadernos de Psicología del Deporte, 16(2), 89-98.

Granero-Gallegos, A., Baena-Extremera, A., Pérez-Quero, F. J., Ortiz Camacho, M. M., y Bracho-Amador, C. (2014). Validación española del Intention to partake in leisure-time physicalactivity. Retos. Nuevas tendencias en Educación Física, Deportes y Recreación, 26, 40-45.

Hagger, M. S., Chatzisarantis, N., Culverhouse, T., y Biddle, S. J. H. (2003). The processes by which perceived autonomy support in physical education promotes leisure time physical activity intentions and behavior: A trans-contextual model. Journal of Educational Psychology, 95, 784-795.

Hui, E. K. P., y Sun, R. C. F. (2010). Chinese children's perceived school satisfaction: The role of contextual and intrapersonal factors. Educational Psychology: An International Journal of Experimental Educational Psychology, 30(2), 155-172.

Informe PISA (2016). Organización para la Cooperación y el Desarrollo Económicos.

Kilpatrick, M., Hebert, E., y Jacobsen, D. (2002). Physical activity motivation. A practitioner's guido to selfdetermination theory. Journal of Physical Education, Recreation and Dance, 74(4), 36-41.

Klomsten, A. T., Marsh, H. W., y Skaalvik, E. M. (2005). Adolescent's perceptions of masculine and feminine values in sport and physical education. A study of gender differences. Sex Roles, 52(9-10), 625-636.

Kofou, I. (2015). What affects motivation in English language learning in the secondary education in Greece? The experience of the 2nd experimental senior high school of Thessaloniki. ICERI2015 Proceedings, 5454-5463.

Krech, P. R., Kulinna, P. H., y Cothran, D. (2010). Development of a short-form version of the Physical Education Classroom Instrument: measuring secondary pupils' disruptive behaviors. Physical Education and Sport Pedagogy, 15(3), 209-225.

Lewis, A. D., Huebner, E. S., Malone, P. S., y Valois, R. F. (2011). Life satisfaction and student engagement in adolescents. Journal of Youth and Adolescence, 40, 249-262. 
Lyons, M. y Huebner, E. (2016) Academic Characteristics of Early Adolescents with Higher Levels of Life Satisfaction. Applied Research Quality Life, 11(3), 757-771.

Ministerio de Educación, Cultura y Deporte. (2013). Pisa 2012 Informe Español. Madrid: Instituto Nacional de Evaluación Educativa.

Moloi, K. C. (2010). How can schools build learning organizations in difficult education contexts? South African Journal of Education, 30(4), 621-633.

Mora, T. (2012). Avoiding disaffection in the mathematics class at secondary school: the role of teacher gender. Revista de Economía Aplicada, 20(60), 89.

Moreno, J. A., Cano, F., González-Cutre, D., Cervelló, E., y Ruiz, L. M. (2009). Flow disposicional en salvamento deportivo: una aproximación desde la teoría de la autodeterminación. Revista de Psicología del Deporte, 18(1), 23-35.

Moreno, J. A., Cervelló, E. M., y González-Cutre, D. (2007). Young athletes’ motivational profiles. Journal of Sports Science and Medicine, 6, 172-179

Moreno, J. A., González-Cutre, D., y Ruiz, L. M. (2009). Self-determined motivation and physical education importance. Human Movement, 10(1), 5-11.

Muela, J. A., García-León, A., Augusto, J. M., y López-Zafra, E. (2010). La enseñanza de la Psicología a través de actividades desde una perspectiva multidisciplinar: aumento de la motivación y rendimiento. European Journal of Education and Psychology, 3(1), 33-44.

OECD. (2013). Education at a Glance 2013: OECD Indicators. Paris: OECD Publishing.

Organización Mundial de la Salud [OMS] (2014). Actividad Física. Disponible en: http://www.who.int/mediacentre/factsheets/fs385/es/

Organización Mundial de la Salud [OMS] (2016). Comisión para acabar con la obesidad infantil. Datos y cifras sobre obesidad infantil. Disponible en: http://www.who.int/end-childhood-obesity/facts/es/

Oviedo, G., Sánchez, J., Castro, R., Calvo, M., Sevilla, J. C., Iglesias, A., y Guerra, M. (2013). Niveles de actividad física en población adolescente: estudio de caso. Retos: nuevas tendencias en educación física, deporte y recreación, 23, 43-47.

Paulsen, M. B. y Feldman, K. A. (2005). The conditional and interaction effects of epistemological beliefs on the self-regulated learning of college students: Motivational strategies research in higher education. Research in Higher Education, 46, 731-768.

Polikhun, N. (2010). Interactive Programs for the Development of Research Activity of Gifted Students. En International Association of Technology, Education and Development (Ed.), Publishes of International Conference of Education, Research and Innovation (pp. 3902-3907). Madrid: IATED.

Reus, L. y Serrano, M. A. (2014). Educación Física y promoción de la salud: estrategias de intervención en la escuela. Retos: nuevas tendencias en educación física, deporte y recreación, 25, 186-191.

Robles, A., Solbes, J., Cantó, J., y Lozano, Ó. R. (2015). Actitudes de los estudiantes hacia la ciencia escolar en el primer ciclo de la Enseñanza Secundaria Obligatoria. REEC: Revista electrónica de enseñanza de las ciencias, 14(3), 361-376.

Rotgans, J. y Schmidt, H. (2011). Situational interest and achievement in the active-learning classroom. Learning and Instruction, 21(1), 58-67.

Ruiz-Juan, F. (2014). Propiedades psicométricas de la versión en español del perceptions of teacher's emphasis on goals questionnaire. Revista mexicana de psicología, 31(2), 164-177.

Smith, J. G. y Suzuki, S. (2015). Embedded blended learning within an Algebra classroom: a multimedia capture experiment. Journal of Computer Assisted Learning, 31(2), 133-147.

Sproule, J., Wang, C. K. J., Morgan, K., McNeill, M., y McMorris, T. (2007). Effects of motivational climate in Singaporean physical education lessons on intrinsic motivation and physical activity intention. Personality and Individual Differences, 43, 1037-1049.

Standage, M., Duda, J. L., y Ntoumanis, N. (2003). A model of contextual motivation in physical education: Using constructs from self-determination and achievement goal theories to predict physical activity intentions. Journal of Educational Psychology, 95, 97-110.

Takakura, M., Wake, N., y Kobayashi, M. (2010). The contextual effect of school satisfaction on health-risk behaviors in Japanese high school students. Journal of School Health, 80(11), 544-551.

Taylor, I. M., Ntoumanis, N., Standage, M., y Spray, C. M. (2010). Motivational Predictors of Physical Education Students' Effort, Exercise Intentions, and Leisure-Time Physical Activity: A Multilevel Linear Growth Analysis. Journal of Sport and Exercise Psychology, 32, 99-120.

Yeung, A., Lau, S., y Nie, Y. (2011). Primary and secondary students’ motivation in learning English: Grade and gender differences. Contemporary Educational Psychology, 36(3), 246-256. 
Satisfacción, motivación y rendimiento académico en estudiantes de Secundaria y Bachillerato: antecedentes, diseño, metodología y propuesta de análisis para un trabajo de investigación

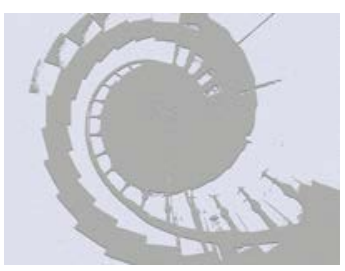

